\title{
Geschlechternormen und Expertise
}

\section{Geschlechterkonstruktionen in psychiatrischen Gerichtsgutachten im Deutschen Kaiserreich 1871-1914}

\author{
Marcus B. Carrier
}

Gender Norms and Expertise. Constructions of Gender in Psychiatrists' Legal Opinions in Imperial Germany, 1871-1914

This article focuses on notions of gender in psychiatrists' expert opinions written for lawsuits during the German Empire, 1871-1914. Four different narratives concerning gender can be identified in these testimonies. On the one hand, the reports describe women and men who did not conform to the gender stereotypes of the time. The psychiatrists found the non-conformist defendants to be of unsound mind. On the other hand, women and men who did conform to the gender stereotypes were also described. In those cases, however, "feminine" women were certified insane, whereas "masculine" men were accused of simulating a mental disease in order to avoid punishment. These findings strengthen basic assumptions of Feminist Epistemology by showing that first, psychiatric ideas of accountability were closely linked to the norm of "masculine" men and second, a double standard was used in assessing the soundness of minds of women and men respectively.

Keywords: Gender, Masculinity, Psychiatry, Lawsuits, Expertise, German Empire

Dieser Artikel beschäftigt sich mit Geschlechterstereotypen in psychiatrischen Gerichtsgutachten während des Deutschen Kaiserreichs 1871-1914. Wie gezeigt werden wird, lassen sich in diesen Gutachten vier auf das Geschlecht bezogene Narrative identifizieren. Einerseits wurden Frauen und Männer beschrieben, die den Geschlechterstereotypen der Zeit nicht entsprachen. Für diese nonkonformen Angeklagten wurde in den hier betrachteten Gutachten die Erklärung zur Unzurechnungsfähigkeit empfohlen. Andererseits wurden aber auch Männer und Frauen beschrieben, die sich konform zu den entsprechenden Stereotypen verhielten. Allerdings wurden in diesen Fällen „weibliche” Frauen weiterhin tendenziell für unzurechnungsfähig erklärt, während „männliche" Männer der Simulation einer Geisteskrankheit bezichtigt wurden, um einer Strafe zu entgehen. Ich werde argumentieren, dass diese Ergebnisse Grundannahmen der Feministischen Epistemologie stärken: sie zeigen erstens, dass psychiatrische Vorstellungen von Zurechnungsfähigkeit eng verbunden waren mit der der Norm des "männlichen" Mannes, und zweitens, dass ein doppelter Standard bei der Beurteilung des Geisteszustands von Männern und Frauen angelegt wurde.

Schlüsse/wörter: Geschlecht, Männlichkeit, Psychiatrie, Gerichtsverhandlungen, Expertise, Deutsches Kaiserreich

Seit inzwischen mehreren Jahren nimmt das Thema wissenschaftlicher Expertise einen wichtigen Platz in der Wissenschaftsforschung ein. Wissenschaftshistorische, -philosophische und -soziologische Ansätze kreisen

NTM-Artikelpreisträger 2016 
dabei insbesondere um Fragen der Glaubwürdigkeit wissenschaftlicher Expertise und der demokratischen Kontrolle derselben. ${ }^{1}$ Besonders in der der anglo-amerikanischen Forschung spielte dabei auch der Spezialfall von Experten vor Gericht eine große Rolle, wahrscheinlich insbesondere deswegen, weil das adversariale Gerichtssystem widerstreitende Expertengutachten in besonderer Weise inszeniert und so die Glaubwürdigkeit der Expertise aus Sicht der Öffentlichkeit besonders in Frage stellt. ${ }^{2}$ Schließlich ist wissenschaftliche Expertise ein wichtiger Teil des Konzepts der Wissensgesellschaft (Stehr 1994: 350-419), das für die historische Forschung insbesondere von Lutz Raphael (1996) und Margit Szöllösi-Janze (2004) mit einem Fokus auf die Humanwissenschaften aufgegriffen wurde.

Im Anschluss an diese Forschungsrichtung hat auch die Geschichte der Kriminologie, beziehungsweise der forensischen Psychiatrie als Subdiziplin derselben, während des Deutschen Kaiserreichs in der jüngeren historischen Forschung verstärkte Aufmerksamkeit erhalten. ${ }^{3}$ Dabei spielte zunächst die Auseinandersetzung mit dem Konzept vom „geborenen Verbrecher" die größte Rolle, das auf den italienischen Anthropologen Cesare Lombroso zurückgeht und sich auf „Degenerationszeichen“ und „Atavismen“ stützte. Allerdings zeigte bereits Mariacarla Gadebusch Bondio (1995), dass Lombrosos Theorie in der Rezeption im Deutschen Kaiserreich nicht einfach übernommen wurde, sondern durchaus umstritten war. Ebenso konstatierte Richard Wetzell (2000), dass biologische Faktoren zur Erklärung von Kriminalität zwar eine Rolle spielten, dabei aber soziale Faktoren keineswegs ausgeklammert wurden. Peter Becker (2002) erweiterte den Blick auf die Kriminologie insofern, als er verschiedene Verbrecherbilder aus philosophischen, anthropologischen, juristischen, literarischen und medizinischen Texten nachzeichnete und rekonstruierte, wie diese sich gegenseitig beeinflussten und überlagerten. Aus einer wissenschaftsund disziplinhistorischen Perspektive im engeren Sinne beschrieb schließlich Silviana Galassi (2004) die Disziplinbildung der Kriminologie als „gebrochene Verwissenschaftlichung“, die sich dadurch auszeichne, dass es der Kriminologie weder gelungen sei, Kriminalität von moralischen Kategorien zu trennen, noch sich erfolgreich als selbstständige Wissenschaft zu institutionalisieren. Urs Germann (2004) schließlich betonte die hohe Komplexität und Kontingenz in der Verbindung von juristischen, psychiatrischen und Verwaltungsdiskursen (er spricht systemtheoretisch von „struktureller Kopplung") sowie Medikalisierungs- und Demedikalisierungstendenzen von Devianz in der deutschsprachigen Schweiz. Dabei bezog er sich nicht nur auf die theoretischen Diskurse, sondern suchte mit dem Fallbeispiel des Kantons Bern auch die Rückbindung an die Praxis. In allen diesen Arbeiten liegt der Fokus größtenteils auf dem Einfluss von Ethnie beziehungsweise „Rasse“ und Klasse auf die Kriminologie; das Geschlecht als soziale Kategorie spielte hingegen kaum eine Rolle. Ausnahmen bilden Christan Müllers 
Dissertation, in der das Thema Geschlecht kurz behandelt wird, allerdings nur in einem Unterkapitel mit der Beschreibung von weiblicher Kriminalität als Sonderfall (Müller 2004: 58-64), und Germanns Dissertation, in der in einem Exkurs auf die so genannte „abnorme Sexualität“ von Männern und Frauen eingegangen wird (Germann 2004: 277-296).

Systematisch wurde die Kategorie Geschlecht in kriminologischen Arbeiten bisher nur von Karsten Uhl (2003) in den Blick genommen. Uhl legte dar, dass bei der Begründung weiblicher Kriminalität innerhalb der Kriminologie zwei Motive regelmäßig auftauchten. Bei dem einen Motiv handelt es sich um die „Übersteigerung der Weiblichkeit“, also die Betonung eines direkten Zusammenhangs zwischen Verbrechen und bestimmten für typisch weiblich gehaltenen Eigenschaften, die bei diesen Verbrecherinnen besonders ausgeprägt gewesen seien (Uhl 2003: 127-138). Bei dem anderen Motiv handelte es sich um die „Vermännlichung“ der Täterinnen, also ein Zusammenhang zwischen Verbrechen und Verhaltensweisen, die nicht als geschlechtskonform markiert waren (Uhl 2003: 138-146). In beiden Fällen basierten diese Beurteilungen auf dem Rückgriff auf Alltagswissen, also gerade nicht auf kriminologischem Spezialwissen (Uhl 2003: 148-152).

Uhl steht damit weitestgehend alleine damit da, psychiatrische Expertise im Deutschen Kaiserreich aus geschlechterhistorischer Sicht zu betrachten. Er konzentrierte sich dabei darauf, theoretische Texte zum Thema der Zurechnungsfähigkeit krimineller Frauen und Frauen im Allgemeinen zu analysieren. Ohne den Stellenwert seiner Studie damit anzuzweifeln, verbleiben dabei allerdings aus Sicht dieses Artikels zwei Aspekte unberücksichtigt, um die diese Perspektive in diesem Aufsatz erweitert werden soll. Es geht dabei aber dezidiert nicht darum, Uhls Ergebnisse infrage zu stellen, sondern sie um diese Aspekte zu ergänzen. Erstens bleibt das Thema der Männlichkeit unberücksichtigt. Die Gefahr einer solchen einseitigen Perspektive besteht darin, dass sie zwar erfolgreich darin ist, Frauen in der Geschichte sichtbar zu machen, dabei jedoch unter Umständen implizit „Frauen“ und "Geschlecht" gleichsetzt. Damit bleibt aber, wie bereits zum Beispiel Ute Frevert (1991) bemerkte, das Geschlecht von Männern in historischen Arbeiten und eben auch bei Uhl weiterhin unsichtbar. Das Problem liegt dann darin, dass eine Geschlechtergeschichte, die Männlichkeit ausblendet, paradoxerweise die Idee reproduziert, Frauen seien doch wieder die Abweichung von der Norm, nämlich von den von ihrem Geschlecht unabhängigen Männern, für die dann die Geschlechtergeschichte nicht zuständig sei. Gerade in der Medizingeschichte jedoch wird dieses einseitige Bild in den letzten Jahren verstärkt infrage gestellt (zum Beispiel Dinges 2007; Benninghaus 2012). Entsprechend werden in dieser Arbeit psychiatrische Gutachten sowohl zu Männern als auch zu Frauen im Hinblick auf geschlechtliche Markierungen und den Rückgriff auf Alltagswis- 
sen der Psychiater über Geschlecht untersucht und verglichen. Dabei wird gezeigt, dass sich eine ähnliche Zweiteilung der Motive für Männer finden lässt, die sich aber nicht in gleicher Weise auf die Beurteilung des Geisteszustandes auswirkten. Wie bei Frauen unterstützte die Zuschreibung von nicht geschlechtskonformen Eigenschaften die Empfehlung der Psychiater, den Angeklagten für unzurechnungsfähig zu erklären. Andererseits unterstützte jedoch die Zuschreibung von als männlich geltenden Eigenschaften in den hier untersuchten Fällen keineswegs die Diagnose einer Geisteskrankheit, sondern untermauerte im Gegenteil den Verdacht der Simulation einer solchen. Wie im Folgenden argumentiert werden wird, spielte diese Unterstützung des Simulationsverdachts für die psychiatrische Expertise insofern eine wichtige Rolle, als ihre Autorität dadurch unterstützt wurde. Durch die so kommunizierte Sicherheit einer unbedingten Erkennung etwaiger Simulationen wurden die Wissensansprüche der Experten grundsätzlich gestärkt und so die Autorität der Expertise untermauert.

Ein solcher Geschlechtervergleich leistet darüber hinaus auch einen Beitrag zur Feministischen Epistemologie, die unter anderem davon ausgeht, dass sich wissenschaftliche Theorien über Menschen in einer männlich dominierten Wissenschaft und Gesellschaft in erster Line vom Mann als Normalfall ausgehen und Frauen an sich schon als Abweichung von der Norm betrachtet werden (so genannte „androzentrische“ Theorien). Damit zusammen hängt auch die Annahme, dass in diesen Fällen bei der Untersuchung von Männern und Frauen mit zweierlei Maß gemessen wird, dass also jeweils dieselben empirischen Befunde bei Frauen und Männern nur aufgrund ihrer Geschlechtszugehörigkeit unterschiedlich gedeutet werden (Anderson 1995). Für diese beiden Annahmen liefert das Ergebnis dieser Arbeit Hinweise in der forensischen Psychiatrie. So wird erklärbar, warum die Zuschreibung von als männlich verstandenen Eigenschaften für eine Simulation und gegen eine echte Geisteskrankheit sprach, wenn gerade solche Männer die Norm darstellen sollten. Die Diagnose einer Geisteskrankheit ist dann - zumindest, wenn sie von geschlechtlichen $\mathrm{Zu}$ schreibungen gestützt werden soll - ausgeschlossen. Auf der anderen Seite wichen Frauen grundsätzlich von dieser Norm ab, wodurch die Feststellung der Unzurechnungsfähigkeit aufgrund der Geschlechtszugehörigkeit bereits bevorzugt wurde. Tatsächlich finden sich - wie gezeigt wird - in den Gutachten zu Frauen durchaus Anmerkungen, die die Zurechnungsfähigkeit von Frauen im Allgemeinen infrage stellten.

Zweitens stehen anders als bei Uhl hier nicht theoretische Texte im Vordergrund, sondern die Praxis der Expertise. Diese Praxis kann nicht mit dem in Lehrbüchern übermittelten Wissen gleichgesetzt werden. Zur Praxis der Expertise gehört in diesem Fall die (klinische) Beobachtung des jeweiligen Angeklagten und die Anfertigung des Gutachtens für das Ge- 
richt. Lehrbücher und theoretisches Wissen hatten darauf selbstverständlich einen Einfluss; eine völlige Fokussierung auf Theorie vernachlässigt allerdings Faktoren der individuellen Fälle und unter Umständen spezifische Anforderungen der Praxis ${ }^{4}$ und riskiert, Diskurse und Praxis ohne Prüfung gleichzusetzen. ${ }^{5}$ Schließlich gilt für psychiatrische Expertise, dass die (Un-) Zurechnungsfähigkeit grundsätzlich keine medizinische, sondern eine juristische Kategorie darstellt. Entsprechend oblag (und obliegt) die Feststellung derselben letztlich dem Gericht und nicht der Psychiatrie. Dies unterscheidet psychiatrische Expertise qualitativ etwa von anderen Gelegenheiten medizinischer oder naturwissenschaftlicher Expertise (Engstrom 2014: 67). Psychiatrische Gutachten können also nicht über die Zurechnungsfähigkeit einer*s Angeklagten entscheiden, sondern dem Gericht nur über eine Diagnose bzw. über die Feststellung einer Simulation eine bestimmte Einstufung empfehlen. Im Zentrum der hier betrachteten psychiatrischen Expertise liegt demzufolge die Kommunikation zwischen Gericht und Experten. Ohne den Stellenwert von theoretischen Texten und Lehrbüchern anzweifeln zu sollen, erscheint es aus dieser Sicht zumindest unvollständig, Gutachten als wichtige direkte Form dieser Kommunikation auszublenden. Entsprechend wurden als Quellen psychiatrische Gerichtsgutachten herangezogen, die im Deutschen Kaiserreich vor dem Ersten Weltkrieg in der Allgemeinen Zeitschrift für Psychiatrie und psychisch gerichtliche Medizin (AZP) veröffentlicht wurden. Neben pragmatischen Gründen der Zugänglichkeit wurden veröffentlichte psychiatrische Gutachten gewählt, weil diese über die Fachveröffentlichung selbst Teil des innerwissenschaftlichen Diskurses waren und so in der Konstellation der psychiatrischen Gerichtsexpertise zur Generierung neuen und spezifischen Expertenwissens beitragen konnten. Dies hat allerdings zur Folge, dass auf dieser Quellenbasis lediglich die Seite der Expertenkommunikation berücksichtigt werden kann. Die Seite des Gerichts wird in den Gutachten höchstens indirekt deutlich, indem zum Beispiel erwähnt wird, ob das Gericht der Empfehlung des Gutachtens folgte. Der Stellenwert, der den Gutachten im jeweils einzelnen Fall von den Gerichten zugemessen wurde, kann hier genauso wenig rekonstruiert werden, wie etwaige kritische Nachfragen in den mündlichen Ausführungen des Gutachters während des Prozesses, da nicht die gesamten Gerichtsakten, sondern eben nur die Gutachten ausgewertet wurden. Darüber hinaus muss der Selektionsprozess der Veröffentlichung beachtet werden. Sicherlich wurden bevorzugt Gutachten mit Kommentar veröffentlicht, wenn die dort behandelten Fälle gerade als nicht typisch verstanden wurden. Dennoch zeigte sich bei der Auswertung aller 86 (identifizierten) Gerichtsgutachten in der Allgemeinen Zeitschrift für Psychiatrie (70 befassten sich mit männlichen, 16 mit weiblichen Angeklagten) in 62 Artikeln starke Ähnlichkeiten in ihren ge- 
schlechtlichen Zuschreibungen. Unter "geschlechtlichen Zuschreibungen“ verstehe ich in diesem Kontext den Rückbezug in Beschreibungen von zu begutachtenden Angeklagten auf Verhaltensweisen und Eigenschaften, die stereotyp mit einem bestimmten Geschlecht in Verbindung gebracht werden. Diese Zuschreibungen können explizit sein, indem zum Beispiel bestimmte Verhaltensweisen und Eigenschaften wörtlich als „unweiblich“ oder „unmännlich“ beschrieben werden, oder implizit, indem sich etwa auf Eigenschaften und Verhaltensweisen bezogen wird, die an anderer Stelle klar dem jeweils anderen Geschlecht zugeordnet wurden. Der impliziten Zuschreibung liegt dabei die Annahme zugrunde, dass Geschlechterstereotypen nicht an die einzelnen Psychiater oder gar an die Profession der Psychiatrie gebunden sind. Es geht hier nicht um spezielle Stereotype, die Psychiater - und nur Psychiater - gehabt hätten, sondern darum, wie sich gesellschaftliche und nicht disziplinär gebundene Annahmen über Geschlecht (hier als "Alltagswissen“ bezeichnet) in der Arbeit der forensischen Psychiatrie zum Ausdruck kam. Methodisch geht es mir also darum, häufig vorkommende Begriffe und inhaltlich ähnliche Beschreibungen, die sich so auf Geschlecht beziehen ließen, aus den Gutachten herauszuarbeiten und in Bezug zur Empfehlung der Gutachter zu stellen. Dabei darf die Empfehlung der Gutachter nicht einfach als eine Funktion der geschlechtlichen Zuschreibungen als einziger Variable verstanden werden; eine solche monokausale Erklärung wäre wohl kaum haltbar oder sinnvoll. Dennoch lässt sich ein Zusammenhang zwischen geschlechtlichen Zuschreibungen und Empfehlung der Gutachter erkennen. Alles was also gezeigt werden soll, ist, dass geschlechtliche Zuschreibungen einen Einfluss hatten (was bereits die These der Feministischen Epistemologie bestätigt), und nicht, dass dieser Einfluss der einzige gewesen wäre.

Vier Narrative ließen sich rekonstruieren, die in diesem Artikel anhand von Fallbeispielen zunächst für Frauen und danach für Männer dargestellt werden. Auf die Frage der etwaigen Repräsentativität über die hier untersuchten Fälle hinaus werde ich an den entsprechenden Stellen für die einzelnen Narrative zurückkommen. Schließlich bleibt noch zu bemerken, dass die hier dargestellte Einheitlichkeit der Narrative keineswegs über theoretische Uneinigkeiten der Psychiater hinwegtäuschen oder gar Differenzen einebnen soll. Es liegt an der Quellengattung der veröffentlichten Gutachten, dem in der Regel eben nicht durch ein zweites Gutachten in derselben Zeitschrift widersprochen wird, dass solche Uneinigkeiten in diesem Artikel keine Rolle spielen. Dies erscheint aber insofern als unproblematisch, als die den hier beschriebenen Zuschreibungen nicht an Theorieschulen gebunden sind, sondern sich gerade für Frauen durch einen direkten Bezug auf Alltagswissen auszeichnen. Gerade in den Geschlechtszuschreibungen fließt also verstärkt ein gesamtgesellschaftlicher 
Geschlechterdiskurs mit ein, was die scheinbare Einhelligkeit der Psychiater in Bezug auf Geschlechtervorstellungen bei aller Differenz in theoretischer Sicht erklärt. Auch in Bezug auf die Narrative erhebt dieser Artikel keinen Anspruch auf Vollständigkeit. Auch wenn in der AZP keine Gutachten gefunden wurden, die den hier dargestellten Mustern systematisch widersprechen, beweist dies natürlich nicht ihre Ausschließlichkeit, insbesondere da hier nur ein sehr spezifischer Ausschnitt des Diskurses untersucht werden konnte, nämlich nur die in genau einer Zeitschrift veröffentlichten Gutachten.

\section{Frauen als Kriminelle}

Im Folgenden wird anhand zweier Fallbeispiele die $\mathrm{Zu}$ - und Beschreibung von als weiblich beziehungsweise männlich konnotierten Eigenschaften bei weiblichen Angeklagten beschrieben. Das erste hier diskutierte Beispiel bezieht sich auf das Motiv der „übersteigerten Weiblichkeit“. Das Motiv tritt besonders stark in einem 1904 veröffentlichten Gutachten des Psychiaters Max Fischer (1862-1940) in den Vordergrund. Fischer war seit 1904 Direktor der psychiatrischen Anstalt Wiesloch bei Heidelberg und hatte vorher in der Anstalt Illenau (1889-1894; 1898-1904) und der Heil- und Pflegeanstalt Emmerdingen (1894-1898) gearbeitet (Kreuter 1996: 341-344). In dem Gutachten findet sich keine Angabe darüber, wann sich der beschriebene Fall genau zugetragen hat.

Die 29 Jahre alte, im sechsten Monat schwangere Landwirtsehefrau N. N. soll an einem Nachmittag in verschiedenen Geschäften Waren im Gesamtwert von 76,70 Mark entwendet haben. Sie habe die Taten erst geleugnet, dann doch zugegeben und Armut als Grund angegeben. Dass sie zunächst geleugnet und Armut als Motiv angegeben habe, stritt sie später ab, was wohl ausreichte, um ihren Geisteszustand zweifelhaft erscheinen zu lassen und eine psychiatrische Begutachtung anzuordnen (Fischer 1904: 312 f.). In der Anstalt verhielt sich die Angeklagte unauffällig, jedoch meinten die Psychiater aus ihrer Familiengeschichte, insbesondere aus dem Alkoholismus des Vaters und wiederkehrenden Anfällen ihrer Schwestern, eine erbliche Belastung ableiten zu können (Fischer 1904: 313 f.). N. N. habe in etwa sechs Jahre dauernder Ehe vier Kinder bekommen, von denen zwei überlebten. Zum Zeitpunkt der Tat war „die Angeklagte wiederum in anderen Umständen, und zwar im sechsten Monat. Die letzte Menstruation war etwa Mitte November, so daß die Zeit ihrer Vergehen mit einem supponierten Menstrualtermin annähernd zusammenfallen würde“ (Fischer 1904: 315). Während ihrer Schwangerschaften sei sie reizbar und 
neige zu Wutanfällen. So einen Wutanfall habe sie auch gehabt, bevor sie am Tag der Tat in die Stadt gefahren sei. Die Schwere dieser Wutanfälle sollen von Schwangerschaft zu Schwangerschaft zugenommen haben (Fischer 1904: $315 \mathrm{f}$.).

In seinem Gutachten verknüpfte Fischer nun diese Beobachtungen mit seinen Ansichten über die „normale“ Frau:

Es ist bekannt, daß das Weib, auch das normale, in der Zeit der Gravidität, abgesehen von den nervösen und körperlichen Begleiterscheinungen, auch psychische Veränderungen eingeht, welche in abnormen sog. Schwangerschaftsgelüsten, ferner in unmotiviertem Stimmungswechsel und in ebenso unmotivierten Antrieben und Impulsen als auch Bewußtseinstrübungen (Dämmerzuständen) ihren Ausdruck finden, wobei die Persönlichkeit als nicht vollständig frei in ihrem Empfinden und Denken, und besonders nicht in ihren Entschlüssen und Handlungen gelten kann - sie ist nicht unbeschränkte Herrin ihres Willens (Fischer 1904: 325 f., Hervorhebung im Original).

Nicht nur der Schwangerschaft wurde hohe Bedeutung für die Beurteilung der Zurechnungsfähigkeit zugesprochen. Auch die Menstruation spielte eine Rolle:

Als besonders beachtenswert erscheint uns im genaueren Verfolg dieses Gesichtspunkts [der Schwangerschaft als auslösendes Moment], daß der Tag der Tat auch mit einem supponierten weiblichen Menstrualtermin zusammenfällt. Die Menstrualtermine sind nun aber in der ganzen forensen [sic] Psychiatrie anerkannt auch beim normalen Weibe, wie viel mehr erst beim psychopathisch veranlagten, als Zeiten, in welchen seine geistige Integrität eine labile ist (Fischer 1904: 327, Hervorhebung im Original).

In der Folge wurde von den Gutachtern empfohlen, N. N. für nicht zurechnungsfähig zu erklären, und das Gericht folgte dieser Empfehlung (Fischer 1904: 329 f.). In diesem Fallbeispiel zeigt sich die enge Verknüpfung von Geisteskrankheit und Weiblichkeit. Die Grenze scheint schmal gewesen zu sein, wie sich insbesondere durch die enge Verbindung zwischen weiblichem Körper und psychischer Gesundheit zeigt. In fast allen Gutachten, die in der Allgemeinen Zeitschrift für Psychiatrie vor dem ersten Weltkrieg zu Frauen veröffentlicht wurden, finden sich mindestens knappe Beschreibungen der Genitaluntersuchungen (zum Beispiel auch in Haßmann 1913; Kelp 1878). Vor allem fällt im Vergleich zu Männern auf, dass diese Untersuchungen anscheinend auch dann erwähnenswert waren, wenn keine Auffälligkeiten festgestellt wurden. Menstruation wurde in fast allen Gutachten über Frauen (zum Beispiel in Kelp 1878; Näcke 
1891), Schwangerschaft immerhin in zwei anderen Gutachten (Kornfeld 1903; Krafft-Ebing 1871) erwähnt. Indem so diese exklusiv weiblichen Körperzustände als auslösende oder verstärkende Faktoren für Geisteskrankheit markiert wurden, wurde die Annahme einer Verbindung, zwischen Verrücktheit und „Natur“ der Frau gestützt. Dies zeigt sich noch einmal deutlich an Fischers Gutachten, in dem er nach der Beurteilung des Einzelfalles dazu übergeht, die allgemeine Zurechnungsfähigkeit von Frauen zu diskutieren:

Ist schon für das gesunde, nicht gravide Weib [...] wegen seiner vom Manne verschiedenen geistigen Verfassung (Mangel an Rechtssinn, Unfähigkeit, Affectstürmen zu widerstehen) strafrechtlich vielfach eine mildere Beurteilung anzustreben, ist ferner das Moment der Menstruation bereits mit vollem Recht als von großem Einflusse auf das Seelenleben des Weibes und für seine Zurechnungsfähigkeit unter Umständen als von eingreifendster Wichtigkeit erkannt worden, so darf hier an dritter Stelle ebenbürtig, wenn nicht in weit bedeutsamerem Maße der gravide Zustand mit seiner totalen Umänderung des somatischen und psychischen Gleichgewichts genannt werden (Fischer 1904: 344).

Wenn Fischer weiter darauf eingeht, dass sich bestimmte körperliche Prozesse von Krankheiten, hier der Hysterie, und der Schwangerschaft ähneln (Fischer 1904: 337-339), erscheint die psychisch kranke Frau als kaum von einer gesunden, aber vielleicht menstruierenden oder schwangeren Frau unterscheidbar. Frauen, so das Motiv dieses Gutachtens, ständen schon durch ihre „Natur“ der Hysterie sehr nahe, die sich letztlich als Übersteigerung der „natürlichen“ Eigenschaften der Frau verstehen lasse.

Der zweite Beispielfall zeigt ein Motiv, in dem gerade die Abwesenheit von als weiblich geltenden Eigenschaften zur Rechtfertigung einer Diagnose der Geisteskrankheit herangezogen wurde. Der Fall wurde 1880 von dem Psychiater Franz Kelp (1809-1892) veröffentlicht und trug sich im Zeitraum zwischen 1856 und 1879 zu. Kelp war von 1858 bis zu seinem Ruhestand 1878 Direktor der oldenburgischen psychiatrischen Anstalt Wehnen, an deren Gründung er als Kreis-Physikus in Delmenhorst (seit 1843) beteiligt war (Kreuter 1996: 701-703). Die Ehefrau des Wirts und Bäckers S. wurde verdächtigt, 1856 ihr jüngstes Kind und einen Untermieter mit Arsen vergiftet zu haben. Außerdem habe sie versucht, sich nach dessen Tod des gesamten Geldes des Untermieters zu bemächtigen. Der Mord an ihrem Kind wurde ihr nicht nachgewiesen, aber sie wurde wegen des Mordes an dem Untermieter, wegen Betrugs und wegen Unterschlagung $1858 \mathrm{zu}$ einer lebenslänglichen Kettenstrafe verurteilt (Kelp 1880: 716 f.). $\mathrm{Zu}$ diesem Zeitpunkt wurde kein Gutachten beantragt. 
Ab 1868 habe Frau S. in der Haft ein merkwürdiges Verhalten gezeigt. So habe sie Halluzinationen und die Angst beschrieben, selbst vergiftet $\mathrm{zu}$ werden. Dies wurde zunächst für eine Simulation gehalten, 1873 wurde sie jedoch zur genaueren Beobachtung in eine Anstalt eingewiesen (Kelp 1880: $718 \mathrm{f}$.). Dabei entstand das hier vorliegende Gutachten. $\mathrm{Zu}$ Beginn dieses Gutachtens wird ausführlich aus den Gerichts- und Gefängnisakten zitiert, deren Quintessenz sich gut im folgenden längeren Absatz widerspiegelt:

Böses Beispiel und schlechte Erziehung im elterlichen Hause mussten früh zeitigen, was die Natur an schlimmen Keimen der Marie S. als Mitgift für's Leben gegeben hatte. Aus einer ungehorsamen Tochter wurde ein diebisches und lügenhaftes Dienstmädchen, bis ihr die Vorsehung einen schwachen Mann zum Ehegatten gab, so schwach, dass er selbst zum Werkzeuge der sich nun ungehindert entfaltenden verbrecherischen Willensthätigkeit der Inquisitin herabsank. Ohne Achtung vor fremden Eigenthum fehlt es ihr nicht minder an Achtung vor den persönlichen Rechten. Nicht nur, dass sie Dritte durch Verläumdung [sic] angreift, sie droht mit Gewahltthätigkeiten. [...] Sie fürchtet sich vor keinem Manne. Ihre Bekanntschaft und Verwandtschaft meidet das gefürchtete boshafte Weib. Sittenlos in ihren Reden, hat sie sich auch insofern des Schmuckes der Weiblichkeit beraubt, von der sie vielleicht nie eine Ahnung hatte (Kelp 1880: $717 \mathrm{f}$.).

In der Anstalt habe sie zwar immer noch ihre Sinnestäuschungen beschrieben, sich aber im ersten Jahr ansonsten "arbeitsam und folgsam“ gezeigt. Erst im zweiten Jahr sei sie durch ihre „rohen Redensarten“ aufgefallen und zeige nun erstmalig auch homosexuelle Neigungen (Kelp 1880: 720 f.). Kelp folgerte, dass Frau S. mindestens seit 1868 geisteskrank sei, also während der Haft nicht simuliert habe, und durchaus der Verdacht bestehen müsse, dass sie auch zum Zeitpunkt der Tat an Sinnestäuschungen gelitten habe (Kelp 1880: 721 f.). Seinen Eindruck von Frau S. fasst er auch noch einmal zum Ende des Gutachtens folgendermaßen zusammen: „Ihr [S.'] ganzes Wesen und Benehmen hatte aber von jeher unweibliches; ihren Mann beherrschte sie vollständig; sie duldete keinen Widerspruch. Sie arbeitete wie ein Mann und gefiel sich in solchen Kräfte erfordernden Beschäftigungen, die nur jenem zukommen“ (Kelp 1880: $722 \mathrm{f}$.).

Insgesamt fällt durch das ganze Gutachten hindurch auf, dass sich die Stellen, in der Frau S. beschrieben wird, stark darauf konzentrieren, wie „weiblich“ - oder eben gerade „unweiblich“ - sie sich verhält und wie sie herrschende Geschlechterbeziehungen durch ihre Sprech- und Handlungsweise untergräbt und hierarchisch umzudrehen scheint. Gegenüber ihrem Mann nahm sie die dominante, den zitierten Beschreibungen zufolge also die eigentlich männliche Rolle ein. Sie wird auch explizit als 
„Virago“ (Kelp 1880: 723) bezeichnet, also als „vermännlichte“ Frau. Dieses nicht geschlechtskonforme Verhalten, das während des Gerichtsprozesses ohne psychiatrisches Gutachten noch eher als ein Indiz für die Schuld von Frau S. gedient hatte, wurde in diesem Gutachten rückblickend pathologisiert. Das Verhalten wurde zu einem Symptom einer erst viel später erkannten Geisteskrankheit erhoben, womit die Schuldfähigkeit nachträglich noch in Zweifel gezogen wurde. 1879 wurde die Strafe von Frau S. in eine lebenslängliche Zuchthausstrafe umgewandelt, also abgemildert (Kelp 1880: 716).

Ähnliche Begründungsmuster finden sich außerdem noch in zwei anderen der veröffentlichten Gutachten (Näcke 1891; Snell 1880). In allen drei Fällen wurden die moralischen Fähigkeiten der Angeklagten als eingeschränkt beurteilt. Insgesamt wirken die Taten geplant und deutlich weniger impulsiv als die Fälle, die dem ersten Beispiel ähneln. Auch wurden keine Zusammenhänge mit der Menstruation oder einer Schwangerschaft festgestellt. Bei allen drei Anklagen handelte es sich um Gewaltverbrechen, die selbst nur in fünf der untersuchten Gutachten, die Frauen behandelten, vorkamen (die anderen sind Haßmann 1913; Roesen 1913). Ein Angriff auf das Leben anderer konnte anscheinend nicht leicht mit den akzeptierten Vorstellungen von Weiblichkeit in Einklang gebracht werden, was auch schon Gustav Aschaffenburg in seinem Lehrbuch Das Verbrechen und seine Bekämpfung feststellte (Aschaffenburg 1903: 129). Ein Vergleich mit den zwei Gutachten, die ebenfalls Gewaltverbrechen behandeln, aber doch eher in das erste beschriebene Motiv passen, also sich gerade nicht auf eine Vermännlichung stützen, erhärtet zusätzlich diesen Verdacht. Bei dem von Walther Haßmann beschriebenen Fall, bei dem eine Frau ihr Kind umbrachte, konnte das Gutachten auf mehrere inzestuöse Beziehungen in den vorherigen Generationen, also auf eine biologische Veranlagung für die Geisteskrankheit hinweisen (Haßmann 1913). Bei Roesen findet sich Heimweh, also „übersteigerte Gefühlsregung“, als Grund für einen versuchten Mord und einen Säureangriff mit schweren Verletzungen eines ansonsten unbescholtenen Mädchens auf ihre Arbeitgeberin (Roesen 1913). Solche Argumentationen wurden anscheinend gebraucht, wenn das allgemeine Verhalten der Frauen im Gegensatz zu den Verbrechen geschlechtskonform war. Der Akt des Gewaltverbrechens war dann eine erklärungsbedürftige Abweichung vom bisherigen Verhalten. Diese Argumentationen wurden aber anscheinend überflüssig, wenn die Zuschreibung männlicher Eigenschaften möglich war.

Was die oben bereits angesprochene Frage der Repräsentativität der Gutachten und damit der Begründung angeht, die durch den Veröffentlichungsprozess verzerrt worden sein könnte, so muss festgehalten werden, dass in Bezug auf die Frauen beide Narrative im Grunde deckungsgleich 
sind mit den Möglichkeiten, die die Studie Uhls erwarten ließ (Uhl 2003: 127-146). Insofern waren die Gutachten in Bezug auf Frauen anscheinend durchaus repräsentativ. Dies mag daran liegen, dass bereits die Tatsache, dass das Verbrechen von einer Frau begangen worden war, als hinreichend außergewöhnlich wahrgenommen wurde, um eine Veröffentlichung zu rechtfertigen. Dies wird auch durch die ebenfalls in der AZP veröffentlichten Statistiken über die in öffentlichen Anstalten begutachteten Angeklagten nahegelegt. Hiernach lag der Anteil von Frauen zwischen 1877 und 1911 nur bei etwa 11 bis 20 Prozent mit sinkender Tendenz (N. N. 1889, 1903, 1905, 1910, 1913).

\section{Männer als Kriminelle}

Ähnlich wie für Frauen, finden sich auch in den Gutachten über Männer zwei Motive, die mit der Geschlechtskonformität des be- und zugeschriebenen Verhaltens zusammenhängen. Um diese zu verdeutlichen, wird auf drei ausgewählte Beispiele zurückgegriffen.

Im ersten Motiv für männliche Angeklagte oder Verdächtigte wurde die Verantwortlichkeit von äußeren Einflüssen betont, für die wiederum diese Männer aufgrund ihres Geisteszustandes besonders anfällig gewesen seien. Dabei ist auffällig, dass diese Anfälligkeit durch die Zuschreibung von weiblichen Eigenschaften gestützt wurde. Dies zeigt sich am ersten hier besprochenen Beispielfall, der 1877 von Eduard Lorent (1809-1886) in der Allgemeinen Zeitschrift für Psychiatrie veröffentlicht wurde und sich 1859 zugetragen hatte (Lorent 1877). Lorent war zwischen 1850 und 1864 Direktor der neueröffneten Irrenabteilung des Allgemeinen Krankenhauses Bremen gewesen, bevor er aus gesundheitlichen Gründen zurücktrat. Er war beteiligt an der Gründung des Vereins für öffentliche Gesundheitspflege in Deutschland und des Vereins der deutschen Irrenärzte (Kreuter 1996: 887). Bei dem durch Lorent und zwei seiner Kollegen begutachteten Angeklagten handelte es sich um den Mechaniker M., der zum Tatzeitpunkt 49 Jahre alt war. Die Anklage wurde erhoben, weil er seinen Arzt angeschossen hatte. Bereits zu Beginn der Darstellung der Tat im Gutachten wurde betont, dass M. vorher nie „erblich krank" gewesen sei und sich außerdem „in seinem früheren Leben stets als ein ruhiger, ordentlicher, besonnener und nüchterner Mann gezeigt" habe (Lorent 1877: 582). Dieser M. erkrankte an einer Infektion des Daumens, deren Behandlung augenscheinlich erforderte, in diesen einzuschneiden. Dies wurde von M.s Hausarzt durchgeführt. Dabei "hatte M. sich sehr empfindlich gezeigt und den Finger weggezogen" (Lorent 1877: 582), wodurch der Schnitt nicht 
richtig durchgeführt worden sei. M. sei in der Folge mit der Behandlung unzufrieden gewesen und konsultierte verschiedene andere Ärzte. Einer von diesen, Dr. N. N., habe wieder einen Einschnitt durchgeführt, allerdings ohne dies M. vorher anzukündigen. „Bei dieser kleinen Operation zeigte M. grosse Empfindlichkeit und wenig Willenskraft" (Lorent 1877: 583). Der diesmal tiefere Schnitt habe M. in große Angst versetzt, da er es für möglich gehalten habe, den Finger aufgrund des Schnittes zu verlieren. Das vorweggenommene Fazit der Gutachter lautet: „Durch die verschiedenen, anscheinend sich widersprechenden Aeusserungen der Aerzte war M. irre geworden" (Lorent 1877: 584). Als sich M. beim nächsten Besuch von Dr. N. N. über die Behandlung beschwert habe, „ermahnte [Dr. N. N.] ihn wegen seines unmännlichen Benehmens und äusserte dabei, ,er betrage sich ja wie ein Kind, das [der Schnitt] sei ja unvermeidlich“" (Lorent 1877: 584). Die Schmerzen seien in den folgenden Tagen schlimmer geworden und Zeugen hätten über Stimmungsschwankungen und abfällige Bemerkungen über Ärzte berichtet. Als Dr. N. N. das nächste Mal kam, schoss M. ihm während der Untersuchung ins Bein. Direkt nach der Tat habe er als Grund angegeben, „er wolle demselben [Dr. N. N.] auch einmal Schmerzen verursachen" (Lorent 1877: 584). Die Untersuchung durch die Ärzte für das Gerichtsgutachten wurde dann folgendermaßen zusammengefasst: „In körperlicher Sphäre erscheint seine [M.s] gesteigerte Sensibilität in grosser Empfindlichkeit gegen Schmerz. Die gesteigerte psychische Sensibilität manifestirte sich in seinem Charakter, im Mangel an Selbstbeherrschung, im unmännlichen Benehmen“ (Lorent 1877: 588).

Die meisten hier untersuchten Gutachten über Männer, die zu dem Schluss kamen, dass der Angeklagte nicht zurechnungsfähig gewesen sei, zeigen ähnliche Begründungsstrategien, die sich folgendermaßen zusammenfassen lassen: Als Auslöser für die Taten werden äußere Einflüsse angegeben, bei denen es sich zum Beispiel um eine Erkrankung des Täters oder um den Einfluss von Alkohol handeln konnte. Diesen äußeren Einflüssen könnten diese Männer aufgrund einer angenommenen vererbten oder einer erworbenen Geisteskrankheit nichts entgegensetzen und zeigen so eine Schwäche des eigenen Willens. Dabei wird deutlich, dass es von großer Bedeutung ist, dass die Angeklagten nicht nur Opfer ihrer Triebe waren, sondern irrational handelten. Der Mangel an Rationalität der Tat scheint ein wichtiger Hinweis auf Geisteskrankheit gewesen zu sein. Wie aber an dem Beispiel explizit deutlich wird, sind gerade dieser Mangel an Rationalität und die erhöhte Sensibilität Eigenschaften, die üblicherweise nicht mit männlichem Verhalten in Verbindung gebracht wurden. In den anderen Fällen wird dieses Verhalten zwar nicht explizit als „unmännlich“ bezeichnet, wie es in den zitierten Stellen des Gutachtens über M. geschah, aber durch Betonung der Irrationalität und der Willensschwäche 
wird dies in einen ähnlichen Kontext gerückt. Dies zeigt sich im Vergleich umso deutlicher, wenn gleichzeitig in Gutachten zu Frauen, gerade Willensschwäche, mangelnde Affektkontrolle und Irrationalität schon als grundsätzliche, „natürliche“ Eigenschaften von Frauen diskutiert wurden (Fischer 1904: 344).

Eine noch gesteigerte Variante desselben Motivs findet sich insbesondere in der Begutachtung homosexueller Männer. Dabei sind diese Fälle zusätzlich interessant, da spätestens seit der Veröffentlichung von Richard von Krafft-Ebings (1840-1902) Psychopathia Sexualis in der ersten Auflage von 1886 Homosexualität selbst Gegenstand psychiatrischer Analyse war und entsprechend verstanden als eine die Urteilskraft einschränkende Krankheit einen möglichen Grund für den Ausschluss der Zurechnungsfähigkeit darstellen konnte. Gleichzeitig stellte der $\$ 175$ des Reichsstrafgesetzbuchs männliche Homosexualität explizit unter Strafe. Homosexualität konnte also im Einzelfall gleichzeitig das Verbrechen und den Grund für die attestierte Unzurechnungsfähigkeit darstellen.

Der hier dazu diskutierte Beispielfall wurde von Richard von KrafftEbing selbst 1901 in der Allgemeinen Zeitschrift für Psychiatrie veröffentlicht (Krafft-Ebing 1901). Krafft-Ebing war Professor für Psychiatrie in Straßburg (1872), und Graz (1873-1889). In Graz war er bis 1885 gleichzeitig Direktor der Irrenanstalt Feldhof bei Graz. Von 1889 bis zu seinem Ruhestand kurz vor seinem Tod 1902 war er Direktor der I. beziehungsweise II. (ab 1892) Psychiatrischen Klinik in Wien (Kreuter 1996: 766-774). In dem von ihm beschriebenen Verfahren ging es um den 34jährigen, verheirateten und ,allgemein geachtete[n] Advokat" und zweifachen Vater X., der sich zwischen 1890 und 1899 in insgesamt 16 Fällen als Vormund von Jungen unter 14 Jahren diesen „unsittlich genähert" haben sollte (KrafftEbing 1901: 545):

Sie [die Fälle] bestanden darin, dass X. [...] die Knaben in sein Bureau berief, wo er immer Stock, Klopf- und Radfahrerpeitsche zur Hand hatte. Er benutzte die geringfügigsten Vorkommnisse zur Züchtigung, liess ihnen die Wahl des Instruments und die Entscheidung, ob sie eine grössere Zahl von Schlägen ad posteriora vestimentis obtecta [auf das bekleidete Gesäß] oder eine kleinere Zahl ad podicem nudum [auf das nackte Gesäß] wollten (Krafft-Ebing 1901: 547).

$X$. sei die Züchtigung auf das nackte Gesäß am liebsten gewesen, und habe es hinterher auch gestreichelt oder es gekniffen. Dabei habe er aber niemals "geschlechtliche Erregung" gezeigt (Krafft-Ebing 1901: 547). Öfter habe er Vorwände gesucht, um die Jungen nackt zu sehen und machte auch Geldgeschenke oder ähnliche Zuwendungen von der Bereitschaft der Jungen abhängig, sich auf das nackte Gesäß schlagen zu lassen. 
Bei der Anamnese wurde festgestellt, dass X. aus einer „geachteten Bürgerfamilie" stammte (Krafft-Ebing 1901: 548), aber sowohl seine Eltern als auch seine drei Geschwister verschiedene psychische Krankheiten aufwiesen. Schon als Kind habe er sich im geschlechtlichen Sinne nur für andere Jungen interessiert, was sich auch nach der Pubertät nicht geändert habe:

Nach absolvirtem Studium war es seine Lieblingsbeschäftigung, an der Erziehung von Knaben sich zu betheiligen. Er bewarb sich förmlich um die Vormundschaft über solche und zeigte in dieser Mission die höchste Hingebung und Aufopferung. In den seltenen Fällen, in welchen er die Vormundschaft über kleine Mädchen übernahm, kümmerte er sich nicht weiter um sie, ebensowenig um Knaben, die das 10. Jahr noch nicht überschritten hatten (Krafft-Ebing 1901: 548).

Geheiratet habe er nicht aus Liebe zu einer Frau, sondern nur, um Kinder haben zu können und außerdem um „seine ihm selbst abnorm erscheinenden [sic] vita sexualis zu saniren“ (Krafft-Ebing 1901: 548). Die Beschreibung der körperlichen Untersuchung beschränkte sich auf: „Penis und Testes auffallend klein. In seinem Aeusseren erscheint X. männlich, jedoch fällt eine mehr an die des Weibes erinnernde Gangart auf" (KrafftEbing 1901: 549). In seinem Charakter wird auf mehrere als Abnormitäten wahrgenommene Eigenschaften hingewiesen. Er habe, so heißt es zum Beispiel, „eine eigenartige, in Denk- und Gefühlsweise verschrobene, sentimentale, weichliche, emotive, optimistische Persönlichkeit" (Krafft-Ebing 1901: 549 f.).

Krafft-Ebing beschrieb X. als „ein[en] Fall von psychischer Entartung“ (Krafft-Ebing 1901: 551). Als Symptome dieser „Entartung“ zählte er auf:

abnorm schwache Entwicklung der Vita sexualis (Hyperaesthesia sexualis) bei zurückgebliebenen Genitalien; fehlende Heterosexualität; als Aequivalent Homosexualität, diese von vornherein dem Unreifen zugewendet - Pädophilia erotica und in perverser Triebrichtung im Sinne des Sadismus (Krafft-Ebing 1901: 551).

Dies alleine reiche allerdings nicht aus, um X. in diesem speziellen Fall auch die Zurechnungsfähigkeit abzusprechen. Hierfür sei relevant, ob X. die Unrechtmäßigkeit seiner Handlungen bewusst war, oder ob er der Ansicht war, nur seine Rolle als Pädagoge zu erfüllen. Hier blieb Krafft-Ebing in seinem Gutachten vage, allerdings legte er Unzurechnungsfähigkeit nahe, denn „[d]ieses Bewusstsein des X. von der sexuell perversen und gesetzlich strafbaren Bedeutung seiner incriminierten Handlungen ist [...] Seitens der Anklage nicht erwiesen“ (Krafft-Ebing 1901: 552). Das Argument, dass Krafft-Ebing hier entwickelte, besagte also, dass die Krankheit die Zurechnungsfähigkeit ausgeschlossen haben könnte, da sie der Grund 
dafür gewesen sein könnte, dass X. seine Handlungen fälschlich als moralisch richtig und pädagogisch notwendig eingestuft habe. Es sei jetzt aber nicht am Gutachter, dies zu beweisen, sondern an der Anklage, diese Möglichkeit auszuschließen. Das Gericht folgte Krafft-Ebings Gutachten nicht, sondern einem zweiten, zuvor eingeholten aber hier nicht veröffentlichten Gutachten, das X. für zurechnungsfähig erklärt hatte, und verurteilte ihn zu zweieinhalb Jahren Haft. Interessant ist, dass Krafft-Ebing in seinen Überlegungen zu dem Fall, die er bei der Veröffentlichung nachträglich seinem Gutachten hinzufügte, davon ausgeht, dass X. unzurechnungsfähig war. Aber selbst, wenn das falsch sei, so Krafft-Ebing, sei X. psychisch krank und gehöre in eine Anstalt, wo „Besserung, ja selbst Heilung“ durchaus möglich sei (Krafft-Ebing 1901: 556).

In den Gutachten, die Homosexualität behandelten (zum Beispiel auch Bischoff 1905: 142; Krafft-Ebing 1890; Richter 1901), fällt auf, dass die Zuschreibung weiblicher Eigenschaften deutlich weitergeht, als an dem ersten Beispiel zu diesem Motiv bereits gezeigt wurde. Nicht nur charakterliche Merkmale werden als „unmännlich“ beschrieben, sondern schon körperlich werden Vergleiche zu Frauen gezogen. Im oben dargestellten Fall zeigt sich dies an der Beschreibung einer "Gangart", die an eine Frau erinnere. Und auch wenn in diesem Beispielfall der Angeklagte ansonsten „männlich“ gewirkt habe, war doch dieser ausdrückliche Verweis auf das Geschlecht des Angeklagten bei der Beschreibung der körperlichen Untersuchung in Gutachten über Männer unüblich.

Das zweite Motiv für männliche Angeklagte setzte sich mit der Simulation einer Geisteskrankheit auseinander. Simulation wurde in diesem Motiv eng mit „männlichen“ Eigenschaften verknüpft. Die Zuschreibung „männlicher" Eigenschaften konnte so also die Feststellung der Simulation einer Geisteskrankheit unterstützen. Dabei ist zu betonen, dass es in diesem Artikel (und in den hierfür herangezogenen Gutachten) nur um eine bestimmte Art von Simulation geht, nämlich um die Simulation einer Geisteskrankheit, um der juristischen Strafe zu entgehen. Wie Sophie Ledebur (2012) ausführt, konnte auch die Simulation bzw. Dissimulation selbst als Symptom für eine Geisteskrankheit verstanden werden, was auch in der Psychiatrie des Kaiserreichs breit diskutiert wurde. Dies ist hier explizit nicht gemeint. Wenn Simulation als Symptom zur Unterstützung einer Diagnose herangezogen wurde, wurden diese Fälle nicht für diesen Abschnitt mitgezählt. Vielmehr geht es hier um so genannte „reine Simulation“, also um den Versuch der Angeklagten der juristischen Strafe durch Vortäuschung einer Geisteskrankheit zu entgehen.

Der dazugehörige Beispielfall spielte sich $1901 \mathrm{ab}$, das Gutachten wurde 1903 vom Psychiater Richard Bolte veröffentlicht (Bolte 1903: 57 f.). Ludwig K. war gelernter Buchdrucker und nach Besuch der Unteroffi- 
ziersschule als Gefreiter nach vier Jahren „wegen militärischer Vergehen und einer Unterschlagung mit dem Führungszeugiss [sic] ,sehr schlecht zur Landwehr entlassen" (Bolte 1903: 57). Dies war nach Informationen der Psychiater offenbar das letzte Mal, dass er einer geregelten Arbeit nachging, denn in den „nächsten 10 Jahre[n] hat er dann anscheinend gewerbsmässig gestohlen und eingebrochen und verschiedene Gefängnissund Zuchthausstrafen durchgemacht" (Bolte 1903: 57). Laut dem vorliegenden Gutachten, kam er diesmal mit dem Gesetz in Konflikt, als er sich am Tag nach Pfingsten 1901 „in einen leeren Laden ein[schlich]“ (Bolte 1903: 57) und dort anscheinend Waren stahl. Während er bei dem ersten Verhör nicht nur Angaben zu seinem Tagesverlauf machen konnte, sondern auch dazu, „wie die bei ihm gefundenen Sachen in seinen Besitz gekommen seien“ (Bolte 1903: 57), habe er aber am nächsten Tag bei seiner Vorführung vor den Richter nicht mehr sprechen können. Auf Empfehlung eines Gerichtsarztes wurde er in ein Krankenhaus verlegt, wo er weiterhin nicht sprach und vorübergehend auch an Inkontinenz gelitten habe. Außerdem habe er sich mit einer Glasscherbe leicht selbst verletzt. „Da man vermuthete, dass er simulire, kam er in die Irrenanstalt zur Beobachtung“ (Bolte 1903: 57). Dort wurden Erkundigungen über ihn eingeholt. „Nach Angabe seiner Frau war er nüchtern, sorgte für sie und liess nur in der ehelichen Treue zu wünschen. Ausser Jähzorn zeigte er keine psychischen Abnormitäten“ (Bolte 1903: 57). Aber auch in der Anstalt habe er meistens nicht reagiert oder geantwortet. Wenn er reagierte, „schien sein Blick etwas scheu und beobachtend" (Bolte 1903: 57). Er wurde daraufhin mit Elektroschocks behandelt, wobei ein anderer Kranker mit ähnlichen Symptomen zum Vergleich herangezogen wurde. „Bei diesem [dem anderen] Kranken traten ganz incoordinirte Bewegungen und zwar nur derjenigen Muskeln auf, die gerade gereizt wurden. Explorand machte dagegen zweckmässige Abwehrbewegungen“ (Bolte 1903: 57 f.). Außerdem habe K. nach den Elektroschocks jeweils Antworten gegeben, die erst falsch gewesen seien, mit der Zeit aber immer genauer wurden. Nachdem die Ärzte in seinem Beisein darüber geredet hätten, dass dieser Zustand vermutlich in drei Tagen vorbei sei, habe er nach Ablauf dieser Frist auch ohne Elektroschocks geantwortet. Nur über die Zeit des Einbruchs und die Zeit in der Anstalt „machte er andauernd falsche Angaben, ordnete zeitlich falsch ein, doch schien dabei ein System zu sein“ (Bolte 1903: 58). Nach diesen Beobachtungen und nachdem die Psychiater also „hinlängliche Proben von seiner [K.s] Verlogenheit zu haben glaubten“ (Bolte 1903: 58), wurde K. aus der Anstalt wieder ins Gefängnis geschickt, um dort auf seinen Prozess zu warten. Die Gutachter erklärten K. zu einem Simulanten, der Richter folgte ihnen und K. wurde zu einer Haftstrafe im Zuchthaus verurteilt. 
Der letzte Absatz des Artikels gibt auch einen Hinweis darauf, wie sich eine solche Simulationsdiagnose auf die Glaubwürdigkeit von Beschwerden in der Haft auswirkte: „Bei Antritt der Zuchthausstrafe, 30. Juli 1901, wollte er [K.] nicht in Einzelhaft, da er es sonst wieder in den Kopf kriege, wurde stumm und verweigerte das Essen. Da aber sofort eine sehr heroische Behandlung (8 Tage Dunkelarrest mit Wasser und Brot) eingeschlagen wurde, gestand er nach einigen Tagen Simulation und zeigte seither durchaus normales Verhalten“" (Bolte 1903: 58). Es war hier anscheinend keine Untersuchung mehr notwendig; die Simulationsdiagnose aus dem Prozess reichte aus, um die Haftbedingungen sofort zu verschärfen. Interessant ist in diesem Zusammenhang ebenfalls, dass in den meisten Simulationsgutachten großer Wert darauf gelegt wurde, zu betonen, wie gut Simulation erkennbar sei. Ohne darüber wirklich Angaben machen zu können - denn dazu wäre Wissen über Fehldiagnosen und nicht nur Wissen über erkannte Simulationen nötig gewesen - wurde hiermit die Sicherheit des wissenschaftlichen Wissens der Gutachter hervorgehoben. Gerade im Kontrast zum ersten Motiv für Männer standen in den Gutachten, die eine Simulation des Angeklagten bescheinigten, Rationalität und berechnendes, überlegtes Verhalten im Vordergrund. Simulation sei gerade deshalb so gut zu erkennen, weil die Patienten, etwa bei falschen Antworten auf Fragen, gewissen Mustern folgten, ein Verhalten, das sie von echten Geisteskrankheiten unterscheide. Simulierte Handlungen wurden also als insbesondere als überlegt und geplant wahrgenommen und dargestellt (zum Beispiel auch in Dietz 1897; Riehm 1908). Im Gegensatz zur Irrationalität und Impulsivität aus dem ersten für Männer diskutierten Beispielfall handelte es sich hierbei um "männliche“ Eigenschaften. Dies wird noch einmal besonders deutlich durch den direkten Vergleich mit dem zweiten für Frauen diskutierten Motiv, in dem genau diese Eigenschaften benutzt wurden, um Frauen ihre Weiblichkeit abzusprechen. Wurde in den Fällen der Unzurechnungsfähigkeit also den Patienten anscheinend die Männlichkeit abgesprochen, zeigt sich hier das Gegenteil. Gerade durch das Vorhandensein "männlicher" Eigenschaften wurde die Diagnose der Simulation und damit die Zurechnungsfähigkeit rhetorisch unterstützt.

Interessant ist, dass in den hier ausgewerteten Gutachten der Verweis auf klar männlich konnotierte psychische Krankheiten völlig fehlte. Dabei existierten solche Krankheiten durchaus; im Allgemeinen schloss also die Zuschreibung männlicher Eigenschaften Geisteskrankheiten bei Männern keineswegs völlig aus. Dass solche Krankheiten existierten zeigt besonders eindrücklich die Geschichte der Militärpsychiatrie und dort besonders Debatten um die „männliche Hysterie“ beziehungsweise besonders der damit thematisch verwandten Neurasthenie (Lengwiler 2000: 77-104) und um 
den „pathologischen Wandertrieb“ (Lengwiler 2000: 137-154). Während solche Diagnosen aber in den hier ausgewerteten Gutachten überhaupt nicht vorkommen, ist die „reine Simulation“ mit 35 von 70 Fällen klar überrepräsentiert. Dies wird besonders auffällig, wenn die theoretische Haltung der Psychiatrie ebenfalls herangezogen wird, zu der Ledebur erklärt, dass „der überwiegende Teil der Psychiater die Meinung [vertrat], dass die sogenannte ,reine Simulation', die bewusste Vortäuschung ohne jegliche geistige Abnormität, nur sehr selten oder auch gar nicht vorkäme“ (Ledebur 2012: 43). Dies legt nahe, dass sie als außergewöhnlich und vermutlich auch als gefährdend für die eigene Autorität angesehen wurde. Die Veröffentlichung dieser Fälle zusammen mit der Bemerkung, dass solche Fälle immer leicht zu erkennen seien, dienten dann der Rückversicherung der Profession, eine sehr hohe Sicherheit der eigenen Diagnose beanspruchen zu können und dem Gericht entsprechend für die Entscheidung der Frage nach der Zurechnungsfähigkeit helfen zu können. Die wenigen Fälle „reiner Simulation“ - so die Botschaft dieser Artikel - konnten eben mit hoher Sicherheit erkannt werden. Die forensischen Psychiater reagierten so auch auf ein Problem ihrer Autorität vor Gericht, nämlich auf die Sorge der Geschworenen und Richter, Simulanten könnten durch eine fehlerhafte psychiatrische Diagnose einer juristischen Strafe entgehen (Engstrom 2014: 67; Ledebur 2012: 40-43). Die männlich konnotierten Eigenschaften, wie etwa „planvolles Vorgehen“, wurden der Unüberlegtheit und Impulsivität, die eher sowohl mit Weiblichkeit als auch mit Wahnsinn in Verbindung gebracht wurden, gegenübergestellt und unterstützten so rhetorisch den Anspruch der Psychiater, solchen Fehlern vorbeugen zu können.

\section{Fazit}

Die angeführten Gutachten der Allgemeinen Zeitschrift für Psychiatrie legen insgesamt den Eindruck nahe, dass es für die Feststellung der $\mathrm{Zu}$ rechnungsfähigkeit von Männern eine wesentliche Rolle spielte, ob sie bei der Untersuchung eher „weibliche“ oder „männliche“ Eigenschaften zeigten. Hingegen scheint die Begutachtung von Frauen im Ergebnis dieser Gutachten insofern unabhängig von der Zuschreibung geschlechtsspezifischer Eigenschaften gewesen zu sein, als sowohl „männliche“ als auch „weibliche“ Eigenschaften die Annahme der Unzurechnungsfähigkeit stützen konnten. Wie bei Männern scheinen die Gutachter geneigt gewesen zu sein, die Frauen bei der Zuschreibung von Eigenschaften des anderen Geschlechts für unzurechnungsfähig zu erklären. Umgekehrt war aber auch die Steigerung von geschlechtskonformem Verhalten bei Frauen (in den 
hier betrachteten Gutachten aber gerade nicht bei Männern) ein Grund für die Diagnose einer psychischen Störung. Bei dem Effekt der Zuschreibung von geschlechtskonformen Attributen in Gutachten bestand also eine deutliche Asymmetrie. Übersteigerte Weiblichkeit war eine deutliche Stütze eines Verdachts auf Unzurechnungsfähigkeit, übersteigerte Männlichkeit hingegen schloss - zumindest in den hier ausgewerteten Gutachten Unzurechnungsfähigkeit fast schon aus und stützte vielmehr einen Simulationsverdacht, im Sinne eines Versuches der bewussten Täuschung des Gerichts und der Psychiater, um einer Strafe zu entgehen.

Im Hinblick auf die Weiblichkeit zeigen sich also die gleichen diskursiven Muster wie bei Uhl. Auch er fand hauptsächlich in theoretischen Texten die Verknüpfung von Unzurechnungsfähigkeit mit einer Übersteigerung der Weiblichkeit auf der einen (Uhl 2003: 127-138) und mit der Zuschreibung männlicher Eigenschaften auf der anderen Seite (Uhl 2003: 138-146). Auch die Vermischung von medizinischem Wissen und gesellschaftlichen Grundannahmen über das Geschlecht hatte Uhl festgestellt (Uhl 2003: 148-152). In diesem Artikel wurde nun, aufbauend auf den Ergebnissen Uhls, zusätzlich das Maskulinitätsstereotyp betrachtet und statt theoretischer Texte hauptsächlich die psychiatrische Begutachtungspraxis berücksichtigt. Interessant ist erstens, dass die Zuschreibung geschlechtsspezifischer Eigenschaften bei Männern nicht mit ausdrücklichem Rückbezug auf Alltagswissen über das Geschlecht stattfand. Nur selten werden bestimmte Eigenschaften explizit als „weiblich“ oder „männlich“ gekennzeichnet; explizite allgemeine Überlegungen zum „normalen“ Mann finden sich überhaupt nicht. Teilweise konnte dieser implizite Gehalt also nur im direkten Vergleich mit der Begutachtung weiblicher Angeklagter identifiziert werden, etwa wenn zur Untermauerung des Simulationsverdachts die gleichen Eigenschaften herangezogen wurden, die bei Frauen zu einer Darstellung der Angeklagten als „vermännlicht" dienten. Gleichzeitig spielten aber zweitens gerade diese unterschwelligen Zuschreibungen anscheinend eine entscheidende Rolle. Unzurechnungsfähige Männer wurden rhetorisch „entmännlicht" beziehungsweise „verweiblicht“. Entsprechungen von Verhalten mit den Männlichkeitsidealen hingegen legten an sich schon Zurechnungsfähigkeit nahe und untermauerten bei auffälligem Verhalten einen Simulationsverdacht. Interessant ist wie gesagt, dass "männliche“ Krankheiten wie Neurasthenie in dieser Auswahl an Gutachten nicht vorkamen. Dies kann als Verzerrung durch die Veröffentlichungspraxis gedeutet werden. Diese Krankheiten waren nicht interessant oder außergewöhnlich genug; Simulation beziehungsweise die Möglichkeit einer falschpositiven Diagnose durch Simulation stellte im Gegensatz zur Neurasthenie potentiell die Expertise und Autorität der Psychiater infrage. Auch könnten sich hier Probleme der Gutachter gezeigt haben, gültige Männ- 
lichkeitsnormen, die für die männlichen Psychiater gleichzeitig Selbstbeschreibung darstellten, mit Geisteskrankheit in Einklang zu bringen. Da alle Gutachten zu Frauen, die während des Untersuchungszeitraumes in der Allgemeinen Zeitschrift für Psychiatrie veröffentlicht wurden, diese für Unzurechnungsfähig erklären, kann an dieser Stelle außerdem keine Aussage darüber getroffen werden, ob geschlechtliche Zuschreibungen dort auch eine Rolle für die Feststellung der Zurechnungsfähigkeit oder einer Simulation spielen konnten. Das Ergebnis dieser Arbeit legt allerdings die (kaum überraschende) Annahme nahe, dass dort (mindestens zusätzlich) andere Zuschreibungen als geschlechtliche für die Begründungen der Gutachter vonnöten waren.

Die Berufung auf Geschlechterstereotype lieferte aber Möglichkeiten, auf Probleme psychiatrischer Autorität vor Gericht zu reagieren. ${ }^{6}$ Erstens waren solche Geschlechterstereotype nicht auf die Psychiatrie beschränkt, sondern durchzogen die Gesellschaft des Kaiserreichs. Der Bezug auf diese Stereotype in den Gutachten kann so dazu beigetragen haben, die Ergebnisse der Gutachten auch für Laien (insbesondere Richter und Geschworene) verständlicher zu machen und das Risiko ihrer Ablehnung zu minimieren. Dies galt auch für die Simulation, die den Laien vor Gericht besondere Sorge bereitete. Zweitens konnte der Bezug auf Geschlechterstereotype nicht nur Uneinigkeiten zwischen Experten und Gericht überbrücken, sondern potentiell auch - zumindest oberflächlich - Uneinigkeiten zwischen verschiedenen psychiatrischen Schulen und Klassifikationssysteme. Germann stellte dazu fest, dass

sich im Hinblick auf „konstitutionelle“ und „einfache Geistesstörungen“ regressive und repetitive Narrative unterscheiden [lassen], welche es erlaubten, biografische und psychopathologische Momente auf unterschiedliche Weise zueinander in Bezug zu setzen. Als Klammer dieser Deutungsmuster und Narrative wie auch als Bezugspunkt zwischen psychiatrischen und juristischen Kriminalitätsdiskursen fungierte die Norm des selbst verantwortlichen und zu einer „sittlichen Selbstführung" fähigen (männlichen) Bürgersubjekts (Germann 2004: 477).

Dieses Ergebnis stützt außerdem zusätzlich zwei Grundannahmen der in der Einleitung angesprochenen Feministischen Epistemologie. Diese geht zum einen davon aus, dass wissenschaftliche Theorien in einem männlich geprägten Wissenschaftssystem den Mann (und als „männlich“ verstandenen Charaktereigenschaften) als Normalfall voraussetzen und Frauen aus diesem Blinkwinkel grundsätzlich schon als Abweichung von dieser Norm erscheinen mussten. An den besprochenen Beispielen zeigt sich dies durch das Narrativ der „übersteigerten Weiblichkeit“, insbesondere wenn diese in Zusammenhang mit Schwangerschaft und Menstruation gesetzt 
wurde. Die so suggerierte Nähe zwischen exklusiv weiblichen Körperzuständen und Geisteskrankheiten kennzeichnete "die“ Frau grundsätzlich als von der Norm abweichend. Ebenso zeigt sich die Normierung der $\mathrm{Zu}-$ rechnungsfähigkeit auf Männer in der Tendenz der Psychiater, als „männlich" beschriebene Männer für zurechnungsfähig und zu Simulanten zu erklären: Zuschreibungen geschlechtskonformer Eigenschaften setzten die begutachteten Männer in die Nähe der gesetzten Norm und erschwerten so potentiell die Diagnose einer Geisteskrankheit und die Erklärung zur Unzurechnungsfähigkeit. Dies hängt auch mit der zweiten hier gestützten Grundannahme der Feministischen Epistemologie zusammen: bei gleichen empirischen Befunden, werden Frauen und Männer aufgrund ihres Geschlechts unterschiedlich beurteilt. In den beiden gerade noch einmal angesprochenen Fällen zeigten sowohl Frauen als auch Männer geschlechtskonformes Verhalten, das jeweils unterschiedlich beurteilt wurde: bei Frauen stützte es Unzurechnungsfähigkeit, bei Männern den Verdacht der Simulation. Auch wenn die Ebene der Geschlechtskonformität verlassen wird und die einzelnen Verhaltensweisen betrachtet werden, zeigt sich eine solche Asymmetrie. Frauen, denen männliche Eigenschaften zugeschrieben wurden, erschienen so etwa keineswegs als näherstehend zur Norm und somit in der Tendenz eher als zurechnungsfähig. Auch vermännlichte Frauen galten den Psychiatern eher als unzurechnungsfähig: bei Männern war „zielgerichtetes" und „rationales“ Verhalten Grund für den Simulationsverdacht, bei Frauen Grundlage für die Absprache der Zurechnungsfähigkeit. Dieses Ergebnis gilt aber - wie nun mehrfach angesprochen - nur für die hier analysierten Fälle. Ob und wie sich „männliche“ Krankheiten, wie die Neurasthenie, hiermit deuten lassen, bleibt eine offene Frage, die sich mit diesen Quellen nicht beantworten lässt.

Für die weitere (wissenschafts-) historische Forschung zum Thema der wissenschaftlichen Expertise demonstriert dies noch einmal den Nutzen, den eine geschlechterhistorische Perspektive haben kann, insbesondere wenn sie nicht nur auf Untersuchungen zur Weiblichkeit beschränkt bleibt. Dabei sind in diesem Aufsatz selbst aber einige Akteure unterbeleuchtet geblieben, woraus entsprechend weitergehende Fragen folgen. Insbesondere war das Geschlecht der Gutachter nicht Teil der Analyse. Die von ihnen in den Gutachten zugrunde gelegten Geschlechtervorstellungen und -normen mussten aber für die meist männlichen Psychiater auch Selbstbeschreibungen darstellen. Ähnliches gilt wohl auch für Richter, Anwälte und Geschworene, die am Ende darüber entscheiden mussten, ob den Gutachten $\mathrm{zu}$ folgen war oder nicht. 


\section{Danksagung}

Dieser Aufsatz basiert auf meiner Bachelorarbeit „Geschlecht, Wahnsinn und Kriminalität. Geschlechterkonstruktionen in psychiatrischen Gutachten im Deutschen Kaiserreich 1871-1914“, die im März 2013 an der Universität Bielefeld angenommen wurde. Ich danke den beiden Betreuern der Arbeit, Vito Gironda und Carsten Reinhardt, für ihre hilfreichen Kommentare und die Ermutigungen, diesen Artikel zu schreiben. Außerdem danke ich Martin Carrier, Gregor Lax, Paul Franke und zwei anonymen Gutachter*innen für ihre Anmerkungen zu früheren Versionen dieses Aufsatzes. Schließlich danke ich der Redaktion der NTM und der DGGMNT für die Verleihung des NTM-Artikelpreises 2016.

\section{Anmerkungen}

1 Zum Beispiel Jasanoff (1990, 1995); Collins \& Evans (2002); Maasen \& Weingart (2005); Hamlin (2008) und Brown (2009).

2 Zum Beispiel Hamlin (1986); Mohr (1993); Golan (1999); Burney (2006) und Watson (2006).

3 Für einen ausgezeichneten Überblick über verschiedene „Wellen“ dieser Forschungsrichtung vgl. auch Engstrom (2014: 63).

4 Zur Praxis in der Psychiatrie zum Beispiel Ledebur (2015: 12-16).

5 Zu dieser Kritik auch Germann (2004: 475).

$6 \mathrm{Zu}$ den folgenden Problemen psychiatrischer Expertise vor Gericht vgl. Engstrom (2014: 67).

\section{Literatur}

Anderson, Elizabeth 1995. Feminist Epistemology: An Interpretation and a Defense. Hypathia (10): 50-84.

Aschaffenburg, Gustav 1903: Das Verbrechen und seine Bekämpfung. Kriminalpsychologie für Mediziner, Juristen und Soziologen, ein Beitrag zur Reform der Strafgesetzgebung. Heidelberg: Carl Winter's Universitätsbuchhandlung.

Becker, Peter 2002. Verderbnis und Entartung. Eine Geschichte der Kriminologie des 19. Jahrhunderts als Diskurs und Praxis. Göttingen: Vandenhoeck \& Ruprecht.

Benninghaus, Christina 2012. Beyond Constructivism? Gender, Medicine and the Early History of Sperm Analysis, Germany 1870-1900. Gender E History (24): 647-676.

Bischoff, Ernst 1905. Simulation von Geistesstörungen. Allgemeine Zeitschrift für Psychiatrie und psychisch-gerichtliche Medizin (62): 124-145.

Bolte, Richard 1903. Ueber einige Fälle von Simulation. Allgemeine Zeitschrift für Psychiatrie und psychisch-gerichtliche Medizin (60): 47-59.

Brown, Mark B. 2009. Science in Democracy. Expertise, Institutions, and Representation. Cambridge: MIT Press.

Burney, Ian A. 2006. Poison, Detection, and the Victorian Imagination. Manchester: Manchester University Press. 
Collins, Harry M. und Robert Evans 2002. The 3rd Waves of Science Studies. Social Studies of Science (32): 235-296.

Dietz, Carl 1897. Simulation von Geistesstörung. Typus: Copie des Kindes. 11/2jährige „Lähmung“. Allgemeine Zeitschrift für Psychiatrie und psychisch-gerichtliche Medizin (53): $1-24$.

Dinges, Martin (Hg.) 2007. Männlichkeit und Gesundheit im historischen Wandel, ca. 1800ca. 2000. Stuttgart: Franz Steiner.

Engstrom, Eric J. 2014. Topographies of Forensic Practice in Imperial Germany. International Journal of Law and Psychiatry (37): 63-70.

Fischer, Max 1904. Schwangerschaft und Diebstahl. Allgemeine Zeitschrift für Psychiatrie und psychisch-gerichtliche Medizin (61): 312-354.

Frevert, Ute 1991. Männergeschichte oder die Suche nach dem „ersten“ Geschlecht. In: Manfred Hettling, Claudia Huerkamp, Paul Nolte und Hans-Walter Schmuhl (Hg.). Was ist Gesellschaftsgeschichte? Positionen, Themen, Analysen. München: C.H. Beck: 31-43.

Gadebusch Bondio, Mariacarla 1995. Die Rezeption der kriminalanthropologischen Theorien von Cesare Lombroso in Deutschland 1880-1914. Husum: Matthiesen.

Galassi, Silviana 2004. Kriminologie im Deutschen Kaiserreich. Geschichte einer gebrochenen Verwissenschaftlichung. Stuttgart: Franz Steiner.

Germann, Urs 2004. Psychiatrie und Strafjustiz. Entstehung, Praxis und Ausdifferenzierung der forensischen Psychiatrie in der deutschsprachigen Schweiz 1850-1950. Zürich: Chronos.

Golan, Tal 1999. The History of Scientific Expert Testimony in the English Courtroom. Science in Context (12): 7-32.

Hamlin, Christopher 1986. Scientific Method and Expert Witnessing: Victorian Perspectives on a Modern Problem. Social Studies of Science (16): 485-513.

Hamlin, Christopher 2008. Third Wave of Science Studies. Toward a History and Philosophy of Expertise. In: Martin Carrier, Don Howard und Janet Kourany (Hg.). The Challenge of the Social and the Pressure of Practice. Science and Values Revisited. Pittsburgh: University of Pittsburgh Press: 160-185.

Haßmann, Walther 1913. Ein Beitrag zur Psychopathologie des Familienmords durch Geisteskranke. Allgemeine Zeitschrift für Psychiatrie und psychisch-gerichtliche Medizin (70): 410-431.

Jasanoff, Sheila 1990. The Fifth Branch. Science Advisors as Policy Makers. Cambridge, MA: Harvard University Press.

Jasanoff, Sheila 1995. Science at the Bar. Law Science and Technology in America. Cambridge, MA: Harvard University Press.

Kelp, Franz 1878. Obergutachten über eine Brandstifterin. Allgemeine Zeitschrift für Psychiatrie und psychisch-gerichtliche Medizin (34): 615-622.

Kelp, Franz 1880. Ueber den Geisteszustand der Ehefrau Catharine Margarethe S-r. Conträre Sexualempfindung. Allgemeine Zeitschrift für Psychiatrie und psychisch-gerichtliche Medizin (36): 716-724.

Kornfeld, Hermann 1903. Gutachten betreffend den Geisteszustand der Frau X. Diebstähle in der Schwangerschaft. Allgemeine Zeitschrift für Psychiatrie und psychisch-gerichtliche Medizin (60): 712-717.

Krafft-Ebing, Richard von 1871. Eine „Mutter im Irrenhaus.“ Allgemeine Zeitschrift für Psychiatrie und psychisch-gerichtliche Medizin (27): 570-585.

Krafft-Ebing, Richard von 1890. Majestätsbeleidigung. Sinnesverwirrung (krankhafte Bewusstlosigkeit) auf Grundlage von Neurasthenie. Allgemeine Zeitschrift für Psychiatrie und psychisch-gerichtliche Medizin (46): 39-44.

Krafft-Ebing, Richard von 1901. Flagellatio puerorum als Ausdruck des larvirten Sadismus eines paedophilen Conträrsexuellen. Fragliche rechtliche Verantwortlichkeit. Allgemeine Zeitschrift für Psychiatrie und psychisch-gerichtliche Medizin (58): 545-557.

Kreuter, Alma 1996. Deutschsprachige Neurologen und Psychiater. Ein biographisch-bibliographisches Lexikon von den Vorläufern bis zur Mitte des 20. Jahrhunderts. 3 Bde. München: Saur.

Ledebur, Sophia 2012. Zur Epistemologie einer Ausschlussdiagnose. Unwissen, Diskurs und Untersuchungstechniken bei Simulation psychischer Erkrankungen. In: Martina Wern- 
li (Hg.). Wissen und Nicht-Wissen in der Klinik. Dynamiken der Psychiatrie um 1900. Bielefeld: transcript: $17-50$.

Ledebur, Sophia 2015. Das Wissen der Anstaltspsychiatrie in der Moderne. Zur Geschichte der Heil- und Pflegeanstalten Am Steinhof in Wien. Wien, Köln und Weimar: böhlau.

Lengwiler, Martin 2000. Zwischen Klinik und Kaserne. Die Geschichte der Militärpsychiatrie in Deutschland und der Schweiz 1870-1914. Zürich: Chronos.

Lorent, Eduard 1877. Gutachten über den Geisteszustand eines den behandelnden Arzt durch einen Schuss verletzenden Kranken. Allgemeine Zeitschrift für Psychiatrie und psychisch-gerichtliche Medizin (33): 582-594.

Maasen, Sabine und Peter Weingart 2005. Democratization of Expertise? Exploring Novel Forms of Scientific Advice in Political Decision-Making. Dordrecht: Springer.

Mohr, James C. 1993. Doctors and the Law. Medical Jurisprudence in Nineteenth-Century America. New York und Oxford: Oxford University Press.

Müller, Christian 2004. Verbrechensbekämpfung im Anstaltsstaat. Psychiatrie, Kriminologie und Strafrechtsreform in Deutschland 1871-1933. Göttingen: Vandenhoeck \& Ruprecht.

N.N. 1889. Statistik. Allgemeine Zeitschrift für Psychiatrie und psychisch-gerichtliche Medizin (45): 449.

N.N. 1903. Statistik. Allgemeine Zeitschrift für Psychiatrie und psychisch-gerichtliche Medi$\operatorname{zin}(60): 637 \mathrm{f}$.

N.N. 1905. Nachweisung. Allgemeine Zeitschrift für Psychiatrie und psychisch-gerichtliche Medizin (62): 123.

N.N. 1910. Nachweisung. Allgemeine Zeitschrift für Psychiatrie und psychisch-gerichtliche Medizin (67): 172.

N.N. 1913. Nachweisung. Allgemeine Zeitschrift für Psychiatrie und psychisch-gerichtliche Medizin (70): 654.

Näcke, Paul 1891. Die Doppelmörderin K. B. Allgemeine Zeitschrift für Psychiatrie und psychisch-gerichtliche Medizin (47): 257-277.

Raphael, Lutz 1996. Die Verwissenschaftlichung des Sozialen als methodische und konzeptionelle Herausforderung für eine Sozialgeschichte des 20. Jahrhunderts. Geschichte und Gesellschaft (22): 165-193.

Richter, Alfred 1901. Zwei im Strafverfahren abgegebene motivirte Gutachten. Allgemeine Zeitschrift für Psychiatrie und psychisch-gerichtliche Medizin (58): 306-336.

Riehm, Walther 1908. Zur Frage der Simulation von Geisteskrankheit. Allgemeine Zeitschrift für Psychiatrie und psychisch-gerichtliche Medizin (65): 28-108.

Roesen 1913. Kasuistischer Beitrag zur Frage der forensisch-psychiatrischen Beurteilung der Heimweh-Verbrecherinnen. Allgemeine Zeitschrift für Psychiatrie und psychischgerichtliche Medizin (70): 975-983.

Stehr, Nico 1994. Arbeit, Eigentum und Wissen. Zur Theorie von Wissensgesellschaften. Frankfurt a. M.: Suhrkamp.

Snell, Ludwig 1880. Ein Gutachten über Zurechnungsfähigkeit. Allgemeine Zeitschrift für Psychiatrie und psychisch-gerichtliche Medizin (36): 450-461.

Szöllösi-Janze, Margit 2004. Wissensgesellschaft in Deutschland: Überlegungen zur Neubestimmung der deutschen Zeitgeschichte über Verwissenschaftlichungsprozesse. Geschichte und Gesellschaft (30): 277-313.

Uhl, Karsten 2003. Das „verbrecherische Weib“. Geschlecht, Verbrechen und Strafen im kriminologischen Diskurs 1800-1945. Münster: LIT.

Watson, Katherine D. 2006. Medical and Chemical Expertise in English Trials for Criminal Poisoning, 1750-1914. Medical History (50): 373-390.

Wetzell, Richard F. 2000. Inventing the Criminal. A History of German Criminlogy, 1880-1945. Chapel Hill: University of North Carolina Press. 
Marcus B. Carrier

Fakultät für Geschichtswissenschaft, Philosophie und Theologie /

Bielefeld Graduate School in History and Sociology

Universität Bielefeld

Postfach 100131

33615 Bielefeld

Deutschland

mcarrier3@uni-bielefeld.de 